\title{
REANÁLISIS DE ASPECTOS CONTROVERSIALES DE LA FONOLOGÍA SEGMENTAL DEL CHEDUNGUN HABLADO EN ALTO BIOBÍO: EL ESTATUS FONÉMICO DEL FONO [y $]^{1}$
}

\section{REANALYSIS OF SEGMENTAL PHONOLOGY CONTROVERSIAL ASPECTS OF THE CHEDUNGUN SPOKEN IN ALTO BIOBIO: PHONEMIC STATUS OF THE PHONE [y]}

\author{
Carolina Salinas Marchant ${ }^{1}$ \\ Gastón Salamanca Gutiérrez \\ Universidad de Concepción ${ }^{1}$ \\ gaston.salamanca@gmail.com
}

\begin{abstract}
Resumen:
En este artículo se determina el estatus fonémico del fono [§], a partir de las distribuciones en las que este ocurre. Los colaboradores son hablantes nativos de mapudungun, seleccionados de acuerdo con su competencia en el vernáculo, pertenecientes a diez localidades de Alto Bío-Bío. La toma de datos se realizó mediante la elicitación de palabras de una lista léxica. El marco de referencia para el análisis de datos considera la tradición de estudios del mapuche en Chile, desde Lenz, hasta los estudios de los últimos tres años, así como también el análisis distribucional de Pike (1947) y Burquest (2009), con aportes de Salas y Poblete (1997) y Salamanca et al (2011). Después de realizado el análisis, se concluye que el fono $[\gamma]$ posee estatus de

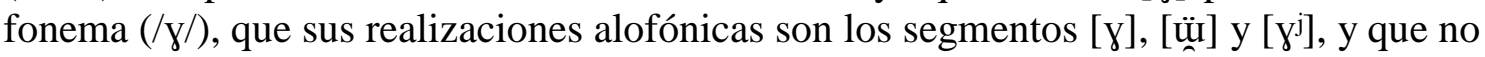
existen mayores diferencias en el comportamiento de este segmento en los subsectores de Queuco y Biobío; ni en los géneros masculino y femenino.
\end{abstract}

Palabras clave: Mapudungun, chedungun, estatus fonémico del fono, Alto del Bío-Bío.

\begin{abstract}
:
This article determines the phonemic status of the phone $[\gamma]$, based on its distribution. The language informants are Mapudungun native speakers, selected according to their competence in the vernacular. They belong to 10 different areas of Alto Bío-Bío. The data collection was done by eliciting words from a lexical list. The framework for data analysis considers the tradition of studies on Mapuche in Chile, from Lenz to the last three years studies. Also, the distributional analysis by Pike (1947) and Burquest (2009), with the contributions of Salas and Poblete (1997) and Salamanca et al (2011). After the analysis was done, it was concluded that the phone $[\gamma]$ has the status of phoneme $(/ \gamma /)$, and its allophonic realizations are the segments $[\gamma],[\ddot{u}] y\left[\gamma^{j}\right]$. It is also concluded that there is no major difference in this segment behavior in the areas of Queuco and Biobio, or in masculine and feminine genders.
\end{abstract}

Keywwords: Mapudungun, chedungun, status of the phone, Alto del Bío-Bío.

Recibido: 1/3/ 2016

Aceptado: 4/12/2016

\footnotetext{
1 Este artículo corresponde a la investigación del proyecto FONDECYT 1131095 (2013-2015). Investigador principal Gastón Salamanca. Co-investigadores Dr. Jaime Soto y Dr. Héctor Painequeo.
} 


\section{Introducción}

El estatus fonémico del fono [४] ha sido motivo de controversia en los estudios fonético-fonológicos del mapudungun, en general, y del chedungun hablado en Alto Bío-Bío, en particular. En este contexto, un objetivo central de este artículo es, precisamente, determinar el estatus de dicho fono en el chedungun hablado en Alto BíoBío. Para ello, se determina la frecuencia con que ocurre y su distribución con respecto a los fonos articulariamente similares $\left[\mathrm{I}^{\mathrm{y}}\right],[\mathrm{w}],[\mathrm{k}],[\mathrm{y}],\left[\mathrm{\gamma}^{\mathrm{j}}\right]$ y [ü $]$.

La metodología considera una revisión de la base de datos del proyecto FONDECYT 1131095. Específicamente, se analiza el corpus fonético-fonológico de muestras de habla del chedungun hablado en Alto Bío-Bío, desarrollando dos macro etapas: (1) determinación de la frecuencia y contextos en que ocurre el fono [४] y (2) análisis distribucional con respecto a otros segmentos articulatoriamente similares.

Uno de los aportes relevantes de esta propuesta es que se re-analiza un foco controversial prominente del mapudungun en general y del chedungun hablado en Alto Biobío en particular, a partir de registros de amplia cobertura -incluyendo, por ejemplo información inédita sobre las localidades pertenecientes al valle del Biobío, y no sólo del valle del Queuco- y realizados con equipamiento técnico de alto estándar.

\section{Marco de Referencia}

\subsection{Estudios fonético-fonológicos prominentes del mapuche hablado en Chile}

Los primeros estudios fonético-fonológicos del mapuche hablado en Chile corresponden a Rodolfo Lenz (1895-1897, Estudio V); sin embargo, no fue hasta 1959, año en que aparece el trabajo de Jorge Suárez, que los estudios del mapuche adoptaron carácter y rigor académico.

Suárez realiza una descripción fonético-articulatoria estandarizada del pehuenche chileno (basada en los datos de Lenz) y realiza un análisis distribucional de los fonos de la lengua. El resultado de este trabajo consiste en un inventario de 27 fonemas: seis vocálicos y veintiún consonánticos. Entrega, además, realizaciones alofónicas y distribución silábica de las mismas. Otro aspecto importante de este análisis es que señala algunas particularidades de la fonología del vernáculo, las que años más tarde serían corroboradas, como: (1) "que en la serie nasal presenta cinco

\footnotetext{
${ }^{2}$ Este apartado se vertebra a partir de las consideraciones contenidas en "Mapuche: Guía bibliográfica", de Salas (1992).
} 
Carolina Salinas Marchant, Gastón Salamanca Gutiérrez. Reanálisis de aspectos controversiales de la fonología segmental del chedungun hablado en Alto BioBío: el estatus fonémico del fono [४]

posiciones fonémicamente diferentes" (1959:178) y (2) que exhibe una alta fluctuación de fonemas, relacionada con la expresión de matices afectivos.

Cuatro años más tarde, se publica una edición, aunque restringida, de la primera descripción fonológica del mapuche hablado en Chile, con datos distintos a los aportados por Lenz, recogidos por Max Echeverría (1963). Al año siguiente, publica la versión definitiva y, en 1965, en colaboración con Heles Contreras, es publicada una versión en inglés, versión acotada a la fonología de la lengua.

De acuerdo con la revisión bibliográfica de la lingüística mapuche realizada por Adalberto Salas (1992), la versión de 1964 es la que ha tenido mayor impacto en los estudios posteriores, sean fonético-fonológicos o morfosintácticos, debido a la metodología empleada y a los numerosos ejemplos proporcionados.

En términos de contenido, Echeverría (1964) presenta: (1) una lista de fonos mapuches descritos articulatoriamente, (2) un análisis fonémico, (3) una lista de fonemas segmentales: seis vocales y veinte consonantes, con sus alófonos, (4) organización de fonemas de acuerdo con rasgos distintivos, (5) fonotaxis de la lengua y (6) un breve comentario de sus rasgos suprasegmentales. Además, proporciona la transcripción fonológica del corpus de investigación.

En 1964, Suárez ofrece una reseña del trabajo de Echeverría (1963), documento en el cual despliega puntos de discrepancia y puntos comunes entre sus trabajos y el de Echeverría.

Entre los puntos de divergencia, Suárez considera que los fonos fricativo velar sonoro [y] y fricativo palatal áfono [S] constituyen fonemas independientes, es decir, no serían variantes de / $\ddot{\mathrm{u}} / \mathrm{y} / \mathrm{t} /$, respectivamente, por lo que su inventario fonológico exhibe veintitrés consonantes.

En 1976 y 1978, Salas entrega una descripción de la fonología del mapuche. En ella, señala sus diferencias con Echeverría (1964) y Suárez (1964).

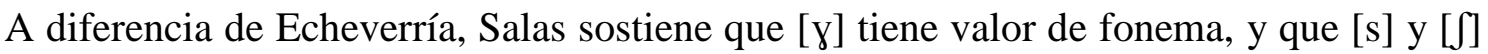
son alófonos de un mismo fonema, aspecto que no consideran sus antecesores. En consecuencia, el inventario fonológico de Salas considera seis vocales y veintiún consonantes.

En 1978, Mary R. Key retoma los estudios de Suárez y desarrolla la noción de fluctuación de fonemas, relacionándola con la evolución diacrónica de la lengua. La autora señala que "la cantidad de fluctuaciones está en relación inversa a la cantidad de pares mínimos: en mapuche hay mucha fluctuación y pocos pares mínimos”. 
En 1981, Daniel Lagos presenta una descripción fonológica del mapudungu(n) hablado en determinadas zonas de la IX región. Su inventario de fonemas considera seis vocales y veinte consonantes, descritas en términos de contraste. Lagos, al igual que Echeverría, considera que $[\gamma]$ no es un fonema. Coincidiendo con Salas, sostiene que [s] y [S] corresponden a un solo fonema. En relación con las vocales, los estudios espectrográficos de Lagos revelan que la sexta vocal es una central de altura media [ə]. Tres años más tarde, el mismo Lagos describe el mapuche hablado en otra zona de la región Araucanía: la comuna de Victoria. De este trabajo, se puede extraer la idea de que, geográficamente, las realizaciones sonoras predominan en el norte y las realizaciones sordas, en el sur. Otro aspecto relevante en este estudio es la idea de que $[\gamma]$ no es fonema, sino un desarrollo de la sexta vocal.

En 1986, aparece una descripción fonológica del huilliche hablado en San Juan de la Costa, X región, realizada por Pilar Álvarez-Santullano. Luego, ese mismo año, publica una segunda versión de su estudio, en la que contrasta el huilliche con el castellano. Ambos trabajos dan cuenta del desmantelamiento del sistema fonológico de este dialecto mapuche.

Cabe destacar que Álvarez-Santullano, al igual que investigadores precedentes, considera una alta fluctuación de fonemas en el vernáculo.

En 1989, Gilberto Sánchez publica "Relatos orales en pewenče chileno". En este artículo, encontramos una descripción del sistema fonológico del chedungun hablado en Alto Bío-Bío. Un aspecto relevante y controversial de esta descripción es que en la variante pehuenche no ocurrirían los fonemas interdentales $/ 1-\mathrm{n}_{x}-\mathrm{t} /$. Otro aspecto problemático es el estatus que el investigador asigna al fono fricativo velar sonoro [४], al cual considera un apoyo consonántico de la sexta vocal.

En 1990, Emilio Rivano publica la primera descripción del vocalismo mapuche desde una perspectiva generativa. El autor propone un conjunto de reglas de transformación de rasgos que daría cuenta de los contrastes vocálicos frecuentes en esta lengua.

En 1997, Gastón Salamanca publica "Fonología del pehuenche hablado en el Alto Bío-Bío", identificando veintisiete fonemas: seis vocales, dieciocho consonantes y tres semiconsonantes. Es preciso consignar que Salamanca considera /j, $\mathrm{\gamma}$, w/ como semiconsonantes correspondientes a las vocales /i, ü, u/. El trabajo refiere, además, aspectos fonotácticos, fluctuación de fonemas y distribución acentual. 
En 2009, Salamanca y Quintrileo publican "El mapuche hablado en Tirúa: Fonemas segmentales, fonotaxis y comparación con otras variedades". Destacan la ocurrencia de veinticuatro fonemas: seis vocales, quince consonantes y tres semiconsonantes. Este artículo, al igual que el de Salamanca (1997), contempla elementos fonotácticos, fluctuación de fonemas y distribución acentual, a la vez que asigna estatus fonémico al fono [४], pero no así a las consonantes interdentales de estatus controversial.

Tres años más tarde, Henríquez y Salamanca (2012) divulgan "Rasgos prominentes de la fonología segmental del chedungun hablado en Alto Bío-Bío". La relevancia de este trabajo radica en que la muestra fue proporcionada por una población escolar, lo que permitió evaluar la vitalidad de la lengua en el nivel fonológico. Entre sus hallazgos destacan la ocurrencia de fonos interdentales $[n, 1, t]^{3}$ con estatus de fonema en Butalelbun, la aparición de [J] como alófono de /t $\int /$, y el estatus fonémico del fono $[\gamma]$.

En 2013, fue publicado en el Journal of The Phonetic Association, "Mapudungu", trabajo desarrollado por Sadowsky, Painequeo, Salamanca y Avelino. Este trabajo incorpora métodos acústicos en el estudio del mapuche, además de seis palatografías para demostrar los fonemas interdentales controversiales. Otro aspecto destacable es que adopta sólo la nomenclatura del AFI.

En el año 2013, aparece también la tesis de pregrado de Daniela Mena, denominada "Frecuencia y distribución de vocales ensordecidas en el chedungun hablado por escolares de Alto Bío-Bío". El trabajo apunta a evidenciar el ensordecimiento de vocales en posición de sílaba final, el que ocurriría en variación libre con sus correspondientes vocales sonoras.

En 2014, Héctor Painequeo presenta un estudio sobre la oposición dental/alveolar, el cual arroja que los pares de fonos oclusivo interdental [t] y oclusivo alveolar [t], nasal interdental [n] y nasal alveolar [n], y lateral interdental [1] y lateral alveolar [1], se realizan en contextos análogos, marcando cambios de significado, de modo que se consideran fonemas diferentes.

Este mismo año aparecen dos nuevas tesis de pregrado relacionadas con la temática que nos ocupa, desarrolladas por Toro y Jiménez, las cuales describen la fonología del chedungun de Alto Bío-Bío en las localidades de Chenqueco y

\footnotetext{
${ }^{3}$ Para que no haya confusión con los segmentos postdentales del español ([t, n, n] 1$]$ ), preferimos utilizar aquí el diacrítico de adelantamiento " " "para indicar la interdentalización .
} 
Chevquelavquen, y Trapa-Trapa y Butalelbun, respectivamente. Estas investigaciones dan cuenta de las realizaciones sonoras de los fonemas /v/ y /ð/, y del estatus fonológico de las consonantes interdentales y del fono [ $\mathrm{\gamma}]$.

En 2014, también se presentan dos tesis de magister: Makarena Sánchez describe la fonología del mapuche hablado en Lonquimay; y Chery Pérez, el mapuche hablado en Curarrehue. Sánchez releva las realizaciones sonoras de /v/ y /ð/; el estatus fonémico de las consonantes interdentales y del fono [४]; y el estatus de alófono de /t $\mathrm{f} /$ del fono [J]. Pérez, por su parte, señala las realizaciones áfonas de $/ \mathrm{f} / \mathrm{y} / \theta /$, y el estatus fonémico de las interdentales controversiales, del fono [ $\mathrm{\gamma}]$ y del fono [ $\left.\int\right]$.

Fuentes (2015) estudia los alófonos de los fonemas /j, w, y, zo v, d/ y sus realizaciones aproximantes en un subsector que sigue el curso del río Bío-Bío, con un enfoque acústico-articulatorio. En relación con el segmento [४], Fuentes concluye en su tesis de magister que es un fonema aproximante dentro del sistema fonológico del chedungun hablado en Alto Bío-Bío, pues los datos que midió de forma estadística dan cuenta de realizaciones marcadamente debilitadas: $83,50 \%$ aproximantes; $13,59 \%$, fricativas; y 2,91\%, otras. En consonancia con sus hallazgos, sugiere el cambio del símbolo [४] por [u].

Como es posible observar, el estatus del fono [y] es un punto controversial en los estudios de la lengua mapuche, en general, y del chedungun en particular, de modo que considerarlo en este artículo constituye un aporte a la disciplina lingüística.

\subsection{Análisis distribucional}

Para conocer la fonología de una lengua, es necesario realizar una serie de observaciones, que van desde la transcripción fonética de los datos recogidos, luego realizar un análisis distribucional y culminar con un acercamiento al sistema fonemático. En términos de Burquest (2009:33), el objetivo de un análisis fonológico es "determinar si esos segmentos fonéticamente similares, en realidad, son la misma unidad fonológica o no".

De acuerdo con el análisis distribucional, podemos consignar tres formas de relación entre los segmentos fonéticamente similares: (1) contraste, (2) distribución complementaria y (3) variación libre.

En la distribución de contraste, los segmentos serán fonemas diferentes, mientras que en la distribución complementaria, los segmentos serán variantes de una misma unidad, es decir, alófonos del mismo fonema. Finalmente, en la variación libre, los 
Carolina Salinas Marchant, Gastón Salamanca Gutiérrez. Reanálisis de aspectos controversiales de la fonología segmental del

chedungun hablado en Alto BioBío: el estatus fonémico del fono [४]

segmentos podrán estar en contraste (en cuyo caso se trata de una neutralización/fluctuación de fonemas) o en alternancia (en cuyo caso también son alófonos).

En relación con los procedimientos necesarios para el análisis de segmentos fónicos, Burquest sostiene que es preciso comenzar con el estudio de palabras cortas, las que presentarán una transcripción detallada. Luego, el analista debe seguir los siguientes pasos: (1) realizar un cuadro de fonos, (2) determinar los segmentos fonéticamente similares, (3) buscar contrastes en pares mínimos o pares mínimos aproximados, (4) determinar distribuciones complementarias, (5) determinar variaciones libres, (6) hacer un cuadro de fonemas y su representación fonémica, y (7) determinar si todos los fonemas de la lengua presentan una distribución completa.

En relación con estos pasos, y siguiendo al mismo Burquest (2009), es importante realizar las siguientes precisiones:

1. Cuadro de fonos: debe realizarse un cuadro articulatorio de cada uno de los fonos, el que debe incluir vocales y consonantes. Es preciso recordar que las semivocales, las nasales y las líquidas pueden realizarse como vocales o consonantes de acuerdo con el contexto.

2. Segmentos fonéticamente similares: los sonidos se comparan de acuerdo con la similitud que puedan presentar. En este punto, es relevante conocer el tipo de procesos que puedan darse en la lengua para interpretar los sonidos. Luego de determinar segmentos fonéticamente similares, es necesario determinar si corresponden a distribuciones de contraste o complementaria.

3. Contraste: se buscarán pares mínimos o pares mínimos aproximados, lo que permitirá establecer que dos segmentos contrastan en contextos idénticos o análogos, es decir, serán fonemas diferentes.

4. Distribución complementaria: si no es posible establecer distribución de contraste entre segmentos que ocurren en contextos similares, es factible escribir una regla alofónica y demostrarla con ejemplos pertinentes.

5. Determinar variaciones libres: si ya se ha probado el contraste y los segmentos varían libremente, se trata de dos fonemas que se neutralizan. $\mathrm{Si}$, en cambio, estos fonos nunca contrastan (no hay cambio de significado), se trata de dos alófonos.

6. Fonemas y representación fonémica: es necesario construir un cuadro de fonemas, sin sus alófonos. Este cuadro debe corresponderse con las intuiciones 
de un hablante nativo de la lengua en estudio, pues sobre esta base se podría elaborar una ortografía. También es adecuado considerar aspectos morfofonológicos para determinar la representación de los fonemas.

7. Distribución de fonemas: en primer lugar, se debe comprobar que todos los fonemas del cuadro presentan una distribución completa. Formas para comprobar:

a) Determinar qué fonemas no aparecen en todos los ambientes posibles.

b) Ver si todas las vocales pueden seguir o no a todas las consonantes del cuadro definido.

c) Establecer la posición del fonema respecto de la sílaba.

Es necesario recordar que el investigador deberá reevaluar las decisiones tomadas durante su análisis, pues cada decisión debe ser verificada y corroborada antes de establecerse como una realidad lingüística.

También es preciso considerar que ni el juicio del observador, ni la intuición del hablante, ni la frecuencia de aparición son, por sí solos, criterios suficientes para tomar decisiones en relación con el análisis de fonemas.

Estas nociones, que tienen su fundamento en las propuestas clásicas de Pike (1947) y Trubetzkoy (1987[1939]) son enriquecidas, entre muchos otros, por las aportaciones de Salas y Poblete (1997) y Salamanca et al. (2011). En el caso de Salas y Poblete (1997), se plantean discusiones interesantes respecto del contraste en ambiente análogo como criterio para asignar estatus fonémico y sobre el fenómeno de la fluctuación de fonemas; mientras que en Salamanca et al. (2011), se elabora un algoritmo para describir los elementos fónicos de una lengua, el que considera la importancia de la distribución de los fonos/fonemas en la sílaba.

Un algoritmo es un conjunto de criterios que empleados de forma ordenada y sucesiva entregarán un producto. El algoritmo propuesto por Salamanca et al. (2011) especifica criterios que permiten definir "desde el estatus fonético de un sonido, hasta el fonológico (unidades funcionales, formas básicas y procesos fonológicos).

Según los autores, para determinar la forma básica de un fonema, se deben considerar los siguientes criterios:

1) Plausibilidad de la direccionalidad del cambio: la forma básica del fonema será aquel fono que permita formular una regla de cambio alofónico con mayor sencillez y claridad. 
2) Simetría del sistema: los fonos que presenten características y contextos opuestos a otros sonidos, incidirán en la selección del representante del fonema, esto para mantener el sistema en equilibrio.

3) Fonotaxis de los fonos en cuestión: la selección del representante del fonema se realizará considerando el fono que presente mayor distribución, es decir, que ocurra en mayor número de contextos.

4) Frecuencia de la realización: la selección por frecuencia solo se utilizará en los casos en que no fuese posible ocupar los otros criterios o persistan las dudas.

\section{Preguntas y Objetivos de Investigación}

La pregunta de investigación que orienta este estudio es: ¿Cuál es el estatus fonémico del fono [ð] en el chedungun hablado en Alto Bío-Bío? De ella, se siguen los siguientes objetivos generales y específicos:

\subsection{Objetivo General}

Determinar el estatus del fono [₹] en el chedungun hablado en Alto Bío-Bío.

\subsection{Objetivos Específicos}

2.2.1. Determinar la distribución en la cual se presenta el fono $[\mathrm{\gamma}]$.

2.2.2. Determinar casos de fluctuación de fonemas donde se presenta el fono [ $\mathrm{\gamma}]$.

\section{Metodología}

La metodología contempla un examen de la base de datos del proyecto FONDECYT 1131095.

Se realizará un análisis fonético-fonológico de muestras de habla del chedungun hablado por sujetos adultos de Alto Bío-Bío; específicamente, se realiza un análisis distribucional del fono $[\gamma]$, con respecto a los fonos articulatoriamente similares $\left[\tau^{\gamma}\right]$, $[w],[k],[y],\left[\gamma^{j}\right]$ y [üu].

3.1. Zona: la recolección de datos se llevó a cabo en diez localidades de Alto BíoBío, ocho de las cuales no habían sido incluidas en estudios previos ${ }^{4}$. Estas son:

\footnotetext{
${ }^{4}$ Los inventarios fonético-fonológicos del chedungun hablado por adultos que existían hasta el momento, se habían realizado a partir de los datos recogidos por Sánchez (1989) y Salamanca (1997), en las comunidades de Cauñicu y Mallla Malla, respectivamente. Henríquez (2013) incluye datos de Cauñicu y Butalelbun, pero del chedungun hablado por escolares.
} 
Butalelbun, Cauñicu, El Avellano, El Barco-Huallali, Malla Malla, Pitril, Ralco Lepoy (subdividido en Chenqueco, Quiñelon, Chevquelavquen) y Trapa Trapa.

Es importante destacar la cobertura de la base de datos, pues permite estudiar en propiedad la fonología del chedungun de Alto Bío-Bío, y no solo de algunas comunidades específicas.

3.2. Sujetos: los colaboradores son 30 hablantes nativos de mapudungun, de entre 20 y 70 años, quienes fueron seleccionados de acuerdo con su competencia en el vernáculo y no presentaban impedimentos físicos ni mentales.

Es preciso destacar que en la selección de los colaboradores participaron el Dr. Héctor Painequeo, co-investigador del proyecto FONDECYT, miembro de la etnia mapuche y hablante nativo de la lengua, y el peñi Horacio Manquepi, quienes aportaron las claves culturales para acercarse a los informantes sin que estos percibieran la investigación como una intromisión más en su cultura, sino, más bien, como un interés genuino por conocer las particularidades de su lengua/variante.

3.3. Instrumentos: se empleó una lista léxica, que tuvo como antecedente la utilizada por Croese (1980). Dicha lista incluyó, además, estímulos que contienen los segmentos problemáticos en las descripciones fonético-fonológicas del área geográfica; entre ellos, pares mínimos aportados por el Dr. Painequeo.

3.4. Sistema de transcripción: la transcripción de las respuestas elicitadas se realizó con los símbolos del AFI, versión 2005, para lo cual se realizaron audiciones pasivas y deliberadas de los datos registrados. Para el análisis auditivo, se empleó la grabadora TASCAM DR-100; y para el apoyo visual, se utilizó una cámara HANDYCAM SONY DCR-DVD108/DVD308/DVD608/DVD708.

3.5. Análisis: básicamente: (1) se cuantifican, en una planilla excel ad hoc, las ocurrencias del fono que nos ocupa y aquellos articulatoriamente similares. La planilla en cuestión informa, además, sobre cada uno de los colaboradores, cada una de las localidades, los dos subsectores (Queuco y Bio-Bío) y el género. En esta presentación, daremos cuenta del comportamiento de este segmento de acuerdo con estas dos últimas variables. (2) se determina si el fono $[\gamma]$ ocurre en distribución contrastante, alternante o complementaria, con respecto a los fonos articulatoriamente similares $\left[\mathrm{l}^{\mathrm{J}}\right],[\mathrm{w}],[\mathrm{k}],[\mathrm{\eta}]$, $\left[\gamma^{\mathrm{j}}\right]$ y $[\ddot{\mathrm{u}}]$. 
Carolina Salinas Marchant, Gastón Salamanca Gutiérrez. Reanálisis de aspectos controversiales de la fonología segmental del chedungun hablado en Alto BioBío: el estatus fonémico del fono [४]

\section{Resultados}

\subsection{El rasgo velar en chedungun y los segmentos articulatoriamente similares a [y].}

En el corpus investigado, se presentan los siguientes fonos que se articulan, total o parcialmente, en el punto de articulación velar: aproximante, retroflejo velarizado, sonoro, oral $\left[\tau^{\mathrm{y}}\right]$, como en [ku.' $\left.\iota^{\mathrm{y} a}\right]$ 'piedra'; aproximante, labiovelar, sonoro, oral $[\mathrm{w}]$, como en [way.'len] 'estrella'; oclusivo, velar, áfono, oral [k], como en ['koi.la]

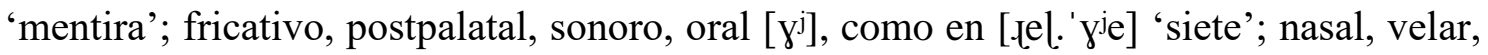
sonoro [y], como en [ya.'pin] 'mujer casada'; y vocoide alta, posterior centralizada, no labializada, asilábica [ü]]. En este contexto, estos fonos se constituyen en fonos articulatoriamente similares a [४]. Son los llamados "segmentos sospechosos" en el Análisis Distribucional.

\subsection{Análisis Distribucional}

Como hemos señalado en el Marco de Referencia, los fonos articulatoriamente similares pueden estar en una relación de contraste, alternancia o complementariedad. Cuando los fonos están en distribución contrastante, las evidencias provienen, en nuestro análisis, de pares submínimos. Sobre esta evidencia, Salas y Poblete (1997:127) señalan:

el par mínimo (contextos idénticos) es una especialización del par submínimo (contextos análogos), de manera que probada la ocurrencia de los sonidos A y B en situación de analogía de contexto, la posibilidad de ocurrencia de A y B en identidad de contexto (par mínimo) queda garantizadamente incluida. Refutada la ocurrencia de A y B en contextos análogos, la posibilidad misma de existencia de un par mínimo entre A y B queda automáticamente excluida.

Por otra parte, con respecto a las fluctuaciones de fonemas, Salas (2006:78) señala:

En mapuche es muy frecuente la llamada fluctuación de fonemas. Simplificadamente, ésta puede ser descrita así: en una palabra dada, por ejemplo, poñi "papa", uno de los sonidos constituyentes, sea $i$, puede ser sustituido por otro sonido, fonéticamente parecido, sea $\ddot{u}$ : ponü. La sustitución es completamente aleatoria, en el sentido de que 1) ocurre sólo en algunas palabras, no en todas, y 2) la sustitución no está asociada a los parámetros normales de variación lingüística, sean geográficos o socioculturales, contextuales o situacionales.

En este artículo se adscribe esta perspectiva.

A continuación, se presenta el estatus que el fono que nos ocupa tiene con respecto a aquellos articulatoriamente similares. 


\subsection{1. $[\gamma]$ y $\left[1^{8}\right]$}

Estos fonos ocurren en distribución contrastante, tal como lo prueban los siguientes pares submínimos:

\begin{tabular}{|c|c|c|}
\hline \#__ & [yal.'yal] 'hongo' & [.tła.'pin] 'vómito’' \\
\hline $\mathrm{V}_{-} \mathrm{V}$ & [na.'yan] 'bajar' & [ma.' '. 'ya] 'liebre' \\
\hline C & [tey.'le] 'siete' & [mə. $\left.\mathrm{l}^{\mathrm{y}} \cdot \mathrm{k}^{\mathrm{j} e}\right]$ 'harina tostada' \\
\hline \# & ['tay] 'greda' & ['tsas'] ' 'pus' \\
\hline
\end{tabular}

En efecto, los fonos en cuestión ocurren en los contextos análogos presentados y se producen cambios de significado. En consecuencia, se presentan en distribución contrastante y pertenecen, por tanto, a fonemas diferentes.

En el caso de $\left[\tau^{\mathrm{\gamma}}\right]$, pertenece al fonema aproximante, retroflejo, sonoro, oral / $\mathrm{t} /$, tal como muestran las siguientes alternancias libres y el hecho de que sea el fono aproximante no velarizado el que ocurre mayoritariamente:

\begin{tabular}{|c|c|c|}
\hline \#__ & [..a.'pin] 'vómito' & [.``a.'pin] 'vómito’ \\
\hline$V_{-}$V & [ma.'.a] 'liebre' & [ma.' 't'a] 'liebre' \\
\hline C & [mə... 'kje] 'harina tostada' & [mə.t. ' $\mathrm{kje}]$ 'harina tostada' \\
\hline _\# & ['tay] 'greda' & ['t'tay] 'greda' \\
\hline
\end{tabular}

Ahora bien, en el corpus se detectan alternancias como las siguientes: [pu.' '. $\left.{ }^{\mathrm{Ya}}\right]$

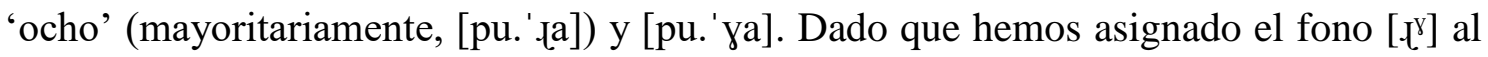
fonema / $/$ /, casos como estos se interpretan como fluctuaciones de (alófonos de) distintos fonemas, donde la forma léxica básica y direccionalidad de la alternancia va en

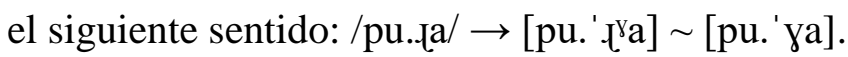

\subsection{2. $[\gamma] y[w]$}

Estos fonos también se encuentran en distribución contrastante, como lo muestran los siguientes enunciados:
\#
[yal.' 'yal] 'hongo'
[wa.jü..'len] 'estrella'
V_V
[na.' 'yan] 'bajar'
[tşa.'wa] 'piel/cuero'

\footnotetext{
${ }^{5}$ Es importante señalar que si bien el ítem 'hongo (del Roble)' se realizó también como [ka.'lal], dos argumentos contribuyen a postular como forma léxica básica /yal.yal/ y no $/ \mathrm{ka} . l \mathrm{lal} /$ : a) la forma [yal.' 'yal] fue mayoritaria en la macrozona y b) en el caso de Alto Bío-Bío, es evidente que las formas sonoras constituyen las formas básicas "por defecto", de modo que partir desde una forma sonora, para derivar una ensordecida, tiene mayor plausibilidad que el caso contrario.
} 
En efecto, los fonos [y] y [w] ocurren en estos contextos análogos y los significados son diferentes. En consecuencia, estos fonos pertenecen a distintos fonemas.

En el caso de $[\mathrm{w}]$, pertenece al fonema /w/, el cual, por distribución complementaria, tiene también a [u] como una de sus realizaciones alofónicas.

\subsection{3. $[\gamma]$ y $[\eta]$}

Al igual que los dos casos anteriores, $[\gamma]$ y $[\mathrm{y}]$ presentan una distribución contrastante, tal como lo comprueban los siguientes contrastes por par submínimo:

\begin{tabular}{|c|c|c|c|}
\hline \#__ & [yal.'yal] 'hongo' & [ya.'pin] & 'mujer casada' \\
\hline $\mathrm{V}_{-} \mathrm{V}$ & [na.'yan] 'bajar' & [ðu.'yun] & 'hablar' \\
\hline $\mathrm{C}$ & [tey.'le] 'siete' & [tson.' 'li] & ‘flaco’ \\
\hline _\# & ['tay] 'greda' & [na'ñn] & 'nuera' \\
\hline
\end{tabular}

Como se ve, los fonos [y] y [y] pertenecen a los fonemas $/ \mathrm{\gamma} / \mathrm{y} / \mathrm{y} /$, respectivamente. Este último también tiene una realización alofónica palatalizada [ $\left.\mathrm{y}^{\mathrm{j}}\right]$.

\subsection{4. $[\gamma]$ y $[k]$}

La distribución de estos fonos también es contrastiva. Así lo muestran los siguientes pares submínimos:

$$
\begin{aligned}
& \text { \#_ [yal.'yal] 'hongo' [ka.'fu] 'verde' } \\
& \text { V_V [na.'yan] 'bajar' [fu.'ka] 'casa' }
\end{aligned}
$$

Como se observa, estos fonos ocurren en contextos análogos y hay cambios de significado. De este modo, los fonos en cuestión pertenecen a distintos fonemas.

El fono $[\mathrm{k}]$ pertenece al fonema $/ \mathrm{k} /$, el cual también tiene como alófono en distribución complementaria al fono oclusivo, postpalatal, áfono [kj].

Considérese ahora el siguiente par de enunciados:

$$
\begin{aligned}
& \text { [pa.'ta.ka] 'cien' } \\
& \text { [pa.'ta. ya] 'cien' }
\end{aligned}
$$

Dado que hemos probado que el fono [k] tiene estatus fonémico, estamos ante un caso de fluctuación de (alófonos de) distintos fonemas, donde la forma léxica y la direccionalidad del proceso de sonorización va en el siguiente sentido:

/pa.ta.ka/ $\rightarrow$ [pa.'ta.ka] [pa.'ta. ya] 


\subsection{5. $[\gamma] y\left[\gamma^{j}\right]$}

A diferencia de los casos precedentes, $[\gamma]$ y $\left[\gamma^{j}\right]$ manifiestan una distribución complementaria y, en consecuencia, pertenecen al mismo fonema. Obsérvense los siguientes enunciados:

$$
\begin{aligned}
& \text { [yal.'yal] 'hongo' } \\
& \text { [na.'yan] 'bajar' } \\
& \text { ['fel.y } \gamma^{\mathrm{j} e} \text { 'siete' } \\
& \text { [ðe.' 'yjin] 'volcán' } \\
& \text { [tse. 'yül] 'treile' }
\end{aligned}
$$

Se observa que el fono palatalizado $\left[\gamma^{j}\right]$ ocurre ante las vocales anteriores $[\mathrm{e}] \mathrm{e}$ [i]; mientras que el fono velar, ocurre ante vocales no anteriores. De este modo, los fonos $[\gamma]$ y $\left[\gamma^{j}\right]$ se complementan $y$, en consecuencia, pertenecen al mismo fonema $(/ \gamma /)$.

\subsection{6. $[\gamma]$ y $[\ddot{u}]$}

En el caso de los fonos [४] y [ü], manifiestan una variación libre restringida. Considérense los siguientes casos:

$$
\begin{aligned}
& \text { \#__ [yal.'yal] 'hongo' } \\
& \text { V_V [na.'yan] 'bajar' } \\
& \text { _C [.еe.'le] 'siete' } \\
& \text { _\# ['tay] 'greda' ['taüu 'greda' }
\end{aligned}
$$

Como se observa, el fono [ü]] sólo ocurre en el postmargen, y en este contexto

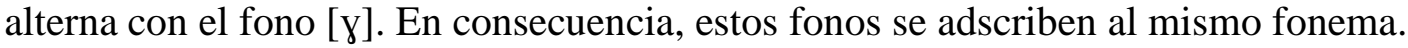
Así, entonces, en virtud de la función demarcativa y opositiva que desempeña el fono [४] respecto de los fonos articulatoriamente similares de la lengua, se puede determinar que constituye un fonema en el chedungun hablado en Alto Bío-Bío. Este fonema posee dos realizaciones alofónicas: el fono $\left[\gamma^{\mathrm{j}}\right]$, que, a diferencia del fono de referencia, ocurre ante vocales anteriores; y el fono [ü]], con el que está en variación libre restringida, en el postmargen silábico.

Dado que el fono fricativo velar $[\gamma]$ ocurre con una mayor distribución y

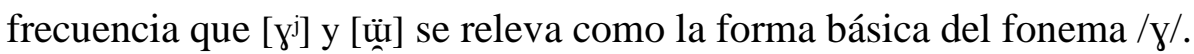

\subsection{Realizaciones del fonema / $/$ / y su frecuencia}

Como se aprecia, hemos asignado estatus fonémico al fono [४]. Ahora, luego de analizar las palabras respecto de las cuales se tenía la expectativa de que se realizarían 
con el segmento que nos ocupa, se obtienen las siguientes realizaciones y sus respectivos porcentajes.

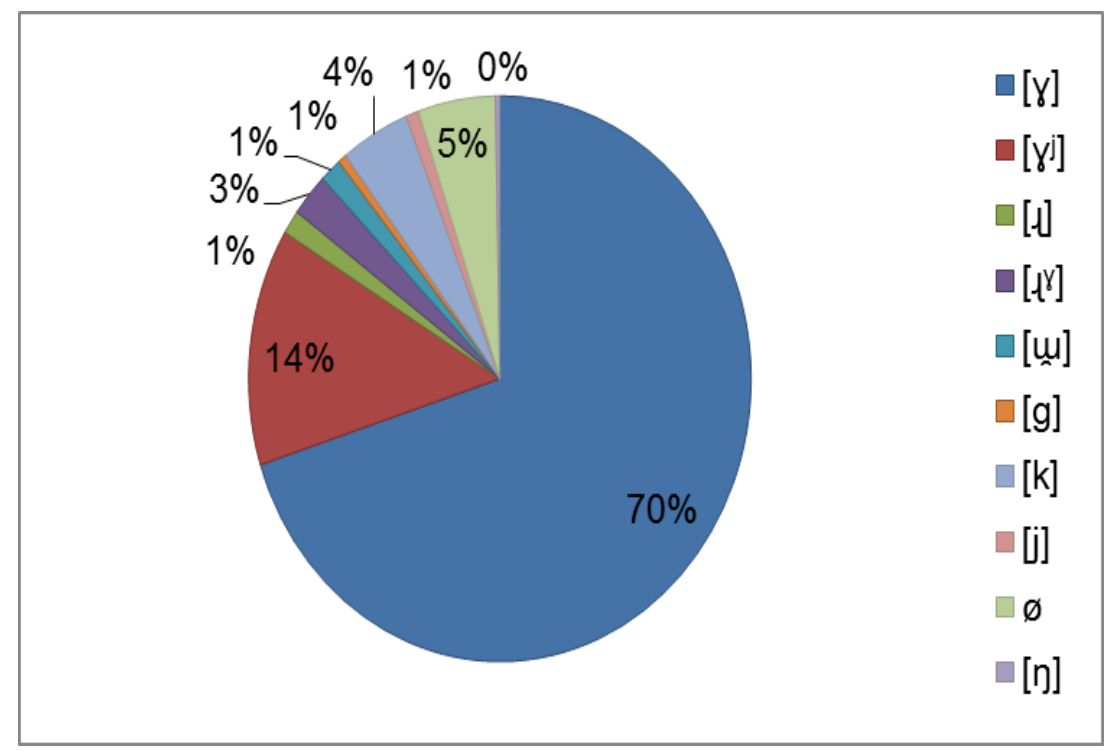

Figura 1. Realizaciones totales del fonema / $\mathrm{\gamma} /$.

De estas realizaciones, como hemos señalado, $[\gamma],\left[\gamma^{j}\right]$, y [ü] constituyen

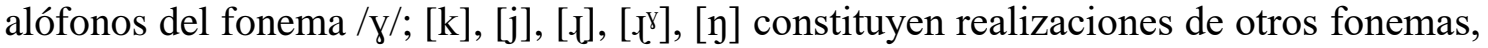
con los cuales el fonema que nos ocupa entra en fluctuación. Por su parte, el fono [g] se interpreta, claramente, como una transferencia del español.

4.4. Comportamiento del fonema $/ \mathrm{y} /$ de acuerdo con las variables género $y$ subsector

\subsubsection{Comportamiento del fonema $/ \gamma /$ de acuerdo con la variable género}

Del total de producciones del fonema $/ \gamma /$ realizadas por informantes de género masculino, los porcentajes mayoritarios corresponden a los alófonos $[\gamma]$, con un $66 \%$; $\left[\gamma^{\mathrm{j}}\right]$, con un $15 \%$; y [ü] $]$, con un $6 \%$. 


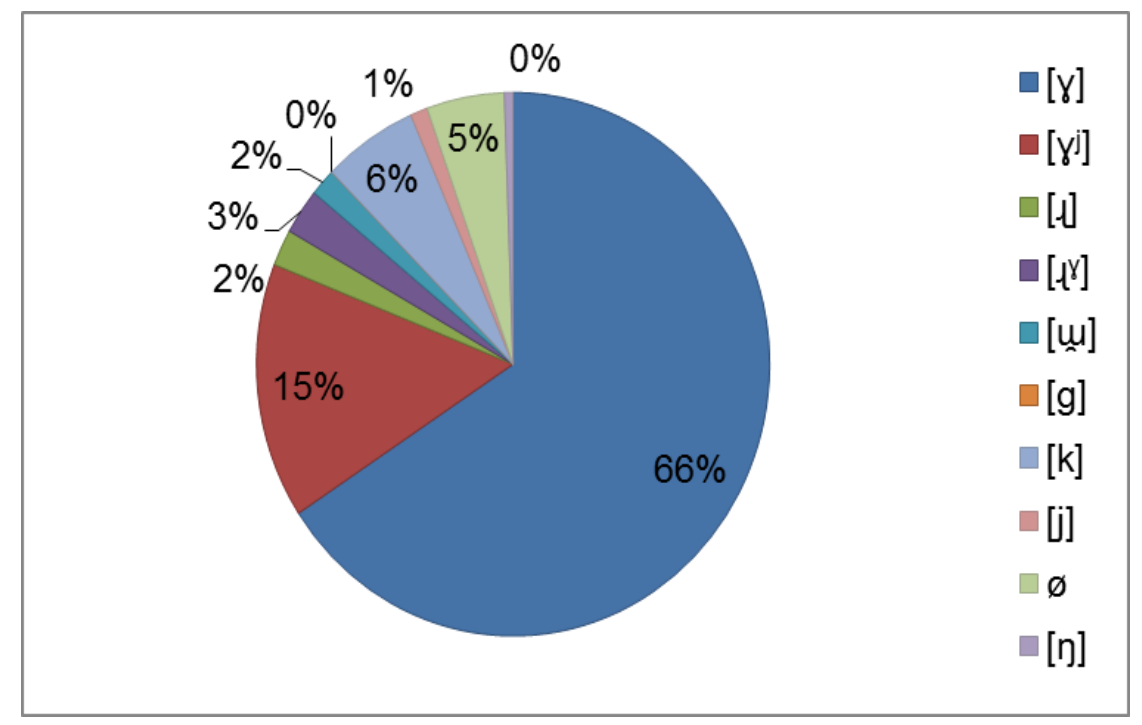

Figura 2. Realizaciones del fonema / $/$ / en hombres.

Tabla 1: Frecuencias absolutas de los alófonos $[\gamma],\left[\gamma^{j}\right]$ y [ü $]$ y otras realizaciones, en hombres.

\begin{tabular}{|c|c|c|}
\hline$/ \mathrm{\gamma} /$ & {$[\mathrm{\gamma}]\left[\mathrm{\gamma}^{\mathrm{j}}\right][\ddot{\mathrm{u}}]$} & Otras \\
\hline 184 & 152 & 32 \\
\hline
\end{tabular}

El porcentaje de las realizaciones alofónicas $[\gamma],\left[\gamma^{j}\right]$ y [ü] , en hombres, es, entonces, de $83 \%$.

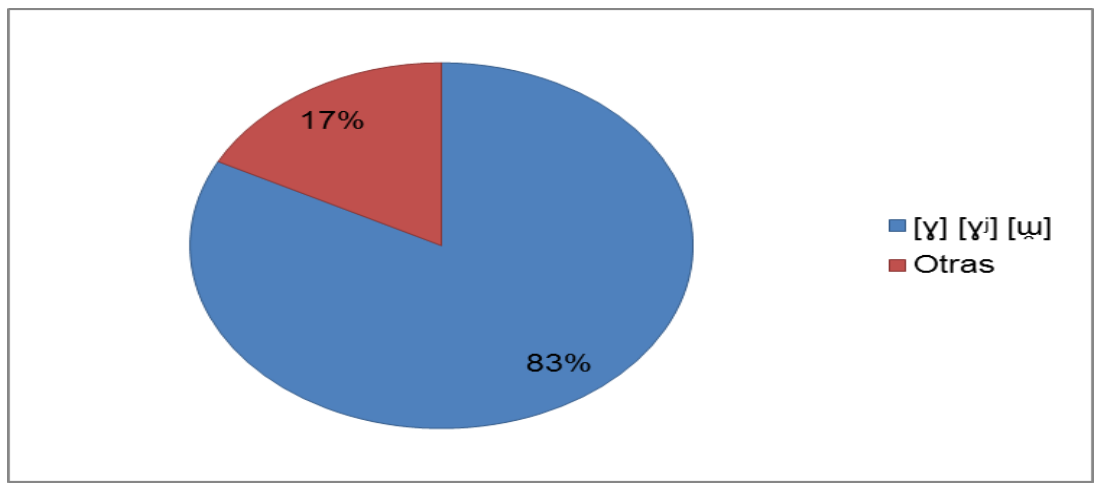

Figura 3. Porcentaje de los alófonos $[\gamma],\left[\gamma^{j}\right]$ y [ü] y otras realizaciones, en hombres.

Del total de producciones de $/ \gamma /$, realizadas por informantes de género femenino, por su parte, los porcentajes mayoritarios corresponden a $[\gamma]$, con un $75 \%$; [ $\left.\gamma^{j}\right]$, con un $12 \%$; y [ø], con un $5 \%$. 


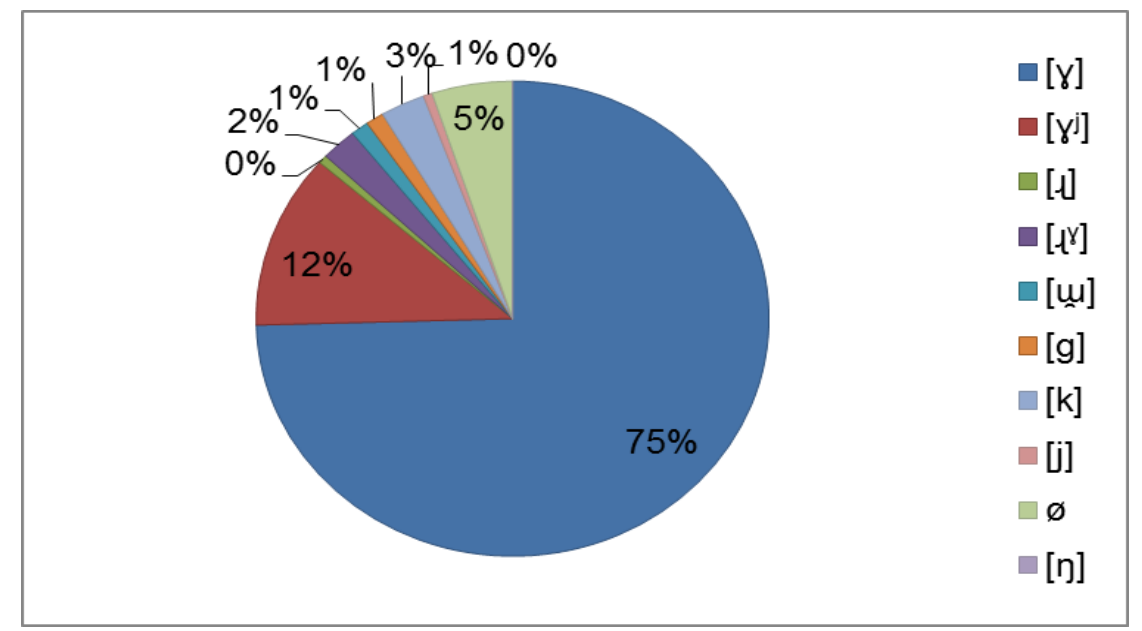

Figura 4. Realizaciones del fonema / $/$ / en mujeres.

La cantidad de realizaciones alofónicas absolutas se presenta en la siguiente tabla.

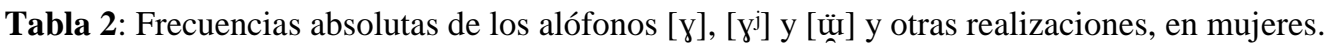

\begin{tabular}{|c|c|c|}
\hline$/ \mathrm{\gamma} /$ & {$[\mathrm{\gamma}]\left[\mathrm{\gamma}^{\mathrm{j}}\right][\ddot{\mathrm{u}}]$} & Otras \\
\hline 177 & 155 & 22 \\
\hline
\end{tabular}

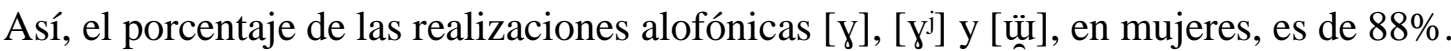

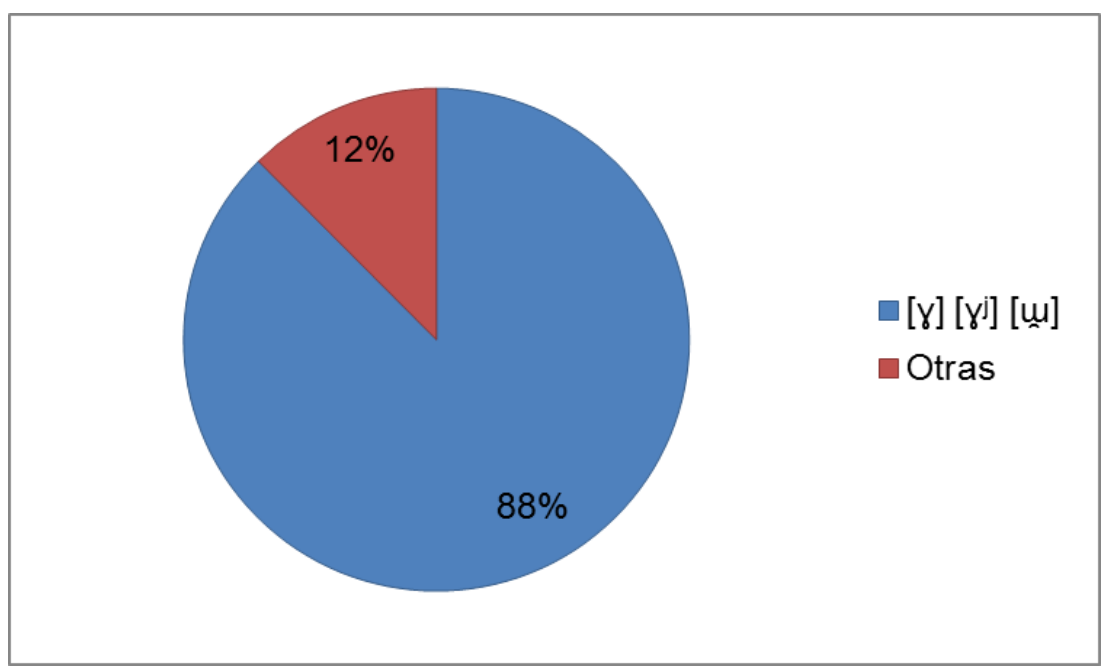

Figura 5. Porcentaje de los alófonos $[\gamma],\left[\gamma^{j}\right]$ y [ü] y otras realizaciones, en mujeres.

Como se observa, el comportamiento del fonema $/ \gamma /$, de acuerdo con la variable género, tiende a la homogeneidad, aunque con un leve predominio en los sujetos de género femenino. 


\subsubsection{Comportamiento del fonema $/ \gamma /$ de acuerdo con la variable subsector}

Del total de producciones del fonema $/ \gamma /$ realizadas por informantes del valle del Queuco, los porcentajes mayoritarios corresponden a $[\gamma]$, con un $75 \%$; [ $\left.\gamma^{\mathrm{j}}\right]$, con un $10 \%$; y $[\varnothing]$, con un $5 \%$.

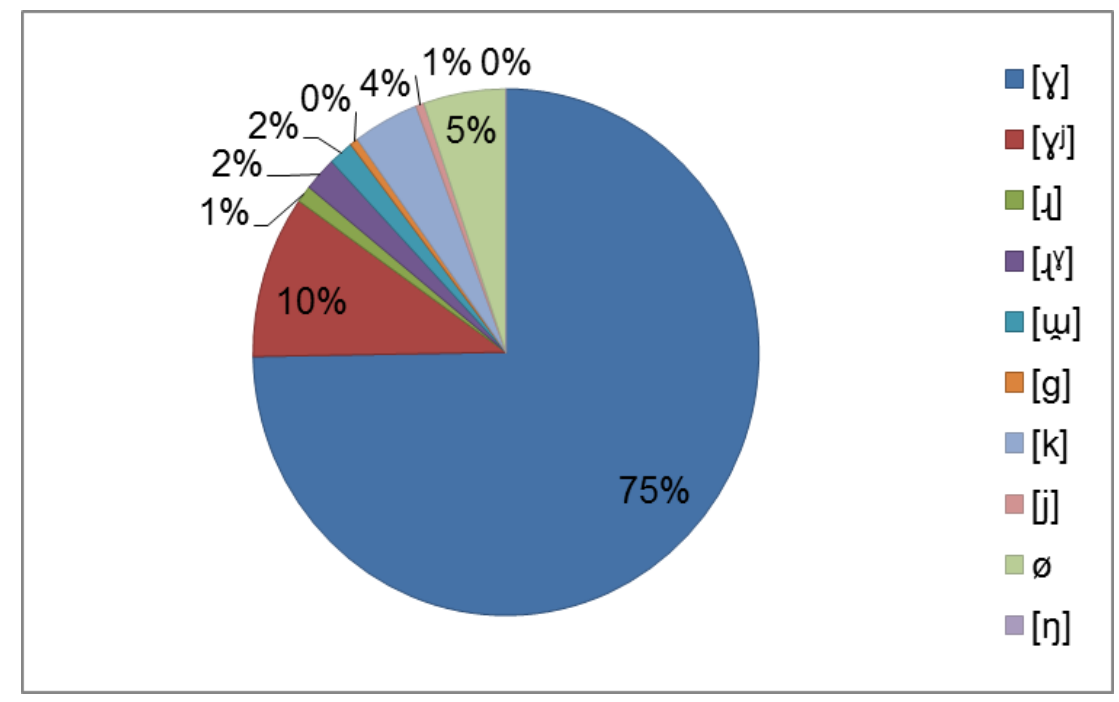

Figura 6. Realizaciones del fonema / $/$ / en Queuco.

La cantidad de realizaciones absolutas de los alófonos de este fonema en Queuco se presenta en la siguiente tabla.

Tabla 3: Frecuencias absolutas de los alófonos $[\gamma],\left[\gamma^{\mathrm{j}}\right]$ y [ü] y otras realizaciones, en Queuco.

\begin{tabular}{|c|c|c|}
\hline$/ \mathrm{\gamma} /$ & {$[\mathrm{\gamma}]\left[\mathrm{\gamma}^{\mathrm{j}]}[\ddot{\mathrm{ü}}]\right.$} & Otras \\
\hline 190 & 164 & 26 \\
\hline
\end{tabular}

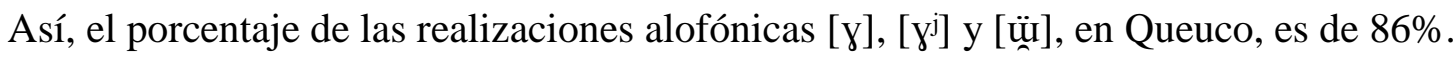




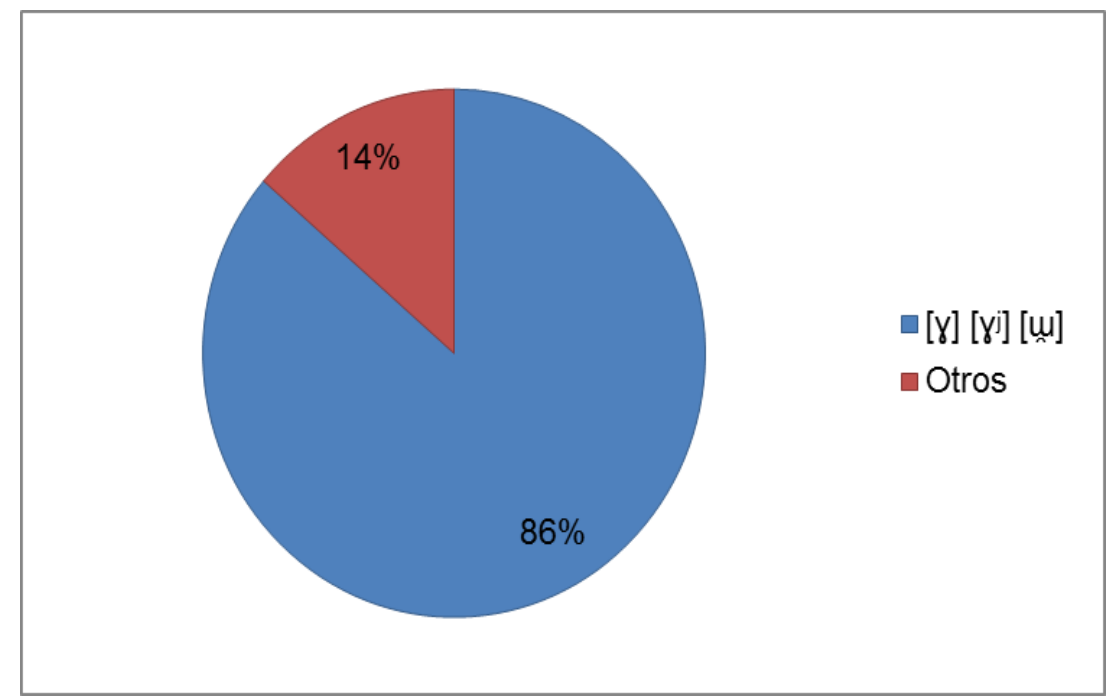

Figura 7. Porcentaje de los alófonos $[\gamma],\left[\gamma^{j}\right]$ y [ü] y otras realizaciones, en Queuco.

Del total de producciones del fonema $/ \gamma /$ realizadas por informantes del valle del Bío-Bío, en tanto, los porcentajes mayoritarios corresponden a $[\gamma]$, con un $64 \%$; $\left[\gamma^{j}\right]$, con un 18\%; y las realizaciones $[\mathrm{k}]$ y $[\varnothing]$, con un $5 \%$ cada una.

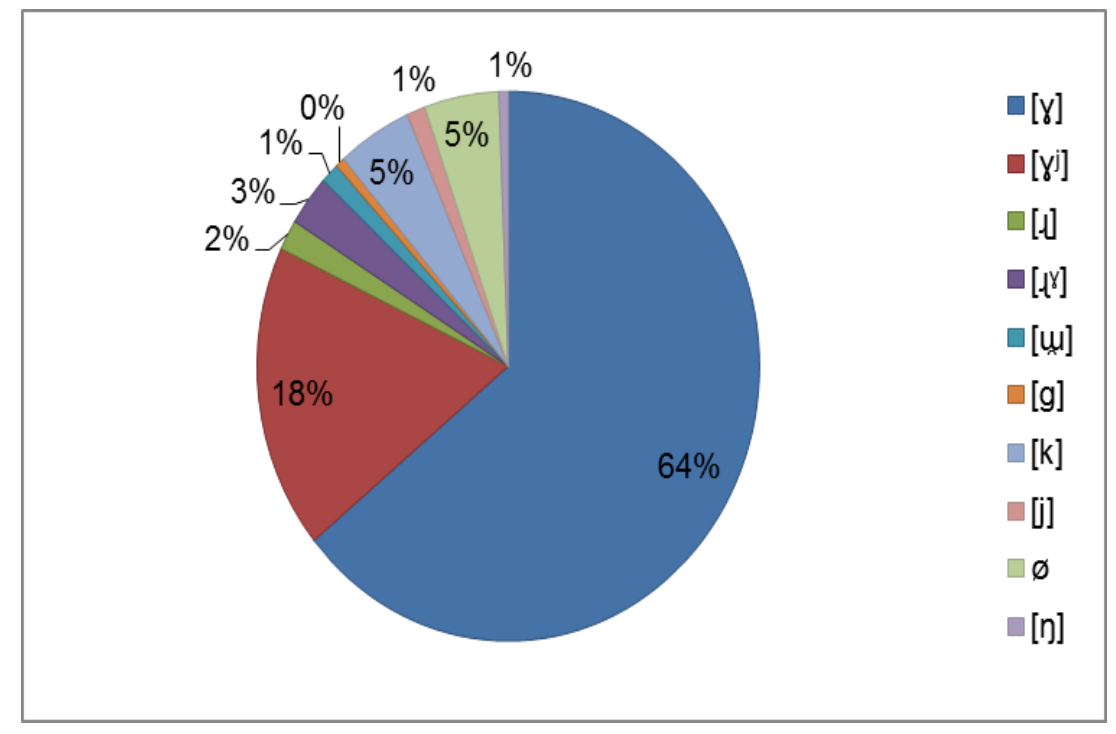

Figura 8. Realizaciones del fonema /y/ en Bío-Bío.

Por su parte, la cantidad de realizaciones absolutas de los alófonos de este fonema en Bío-Bío se presenta en la siguiente tabla. 


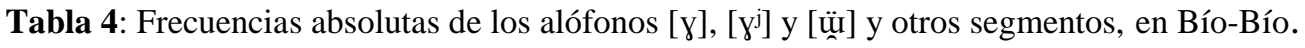

\begin{tabular}{|c|c|c|}
\hline$/ \mathrm{\gamma} /$ & {$[\mathrm{\gamma}]\left[\mathrm{\gamma}^{\mathrm{j}]}[\ddot{\mathrm{u}} \mathrm{i}]\right.$} & Otras \\
\hline 167 & 139 & 28 \\
\hline
\end{tabular}

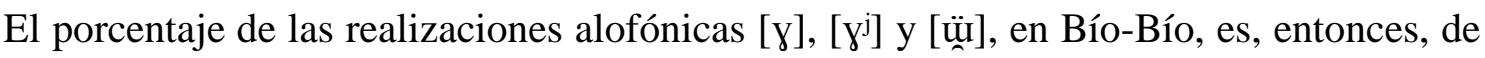
$83 \%$.

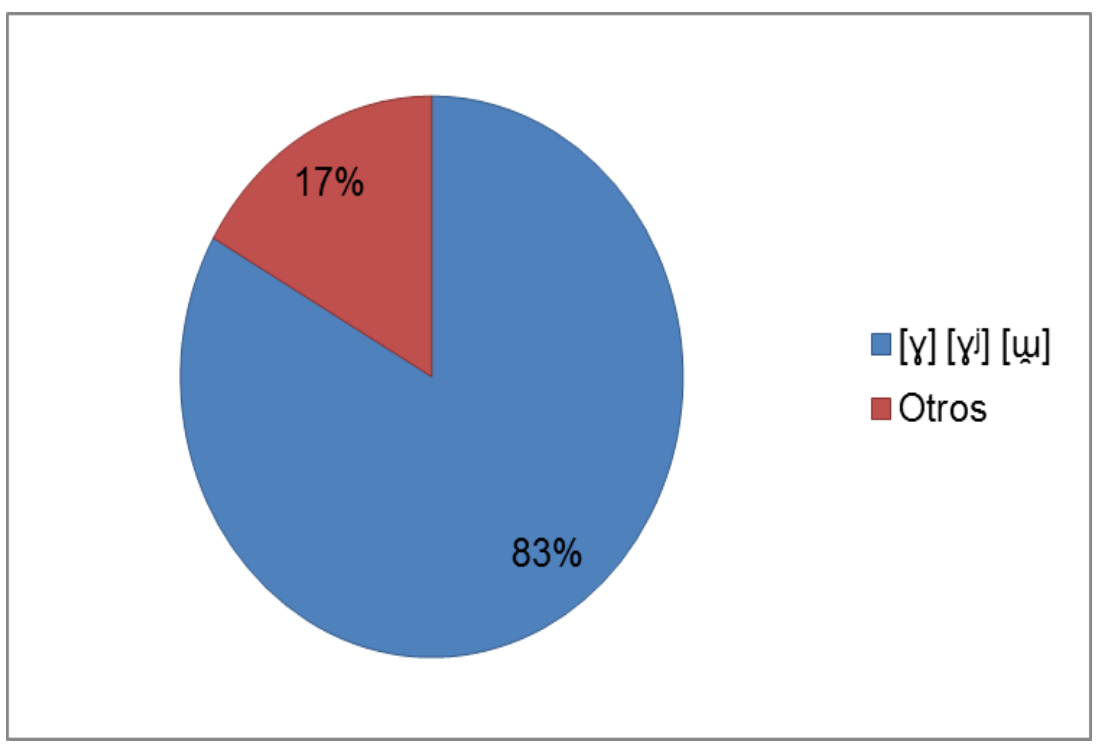

Figura 9. Porcentaje de los alófonos $[\gamma],\left[\gamma^{\mathrm{j}}\right]$ y [ŭ] y otras realizaciones, en Bío-Bío.

Como se observa, en el comportamiento de este fonema de acuerdo con la variable subsector, también se presenta una alta tendencia a la homogeneidad.

\section{Conclusión}

Hemos podido determinar que el fono $[\gamma]$ presenta distribución de contraste por contexto análogo con $\left[\mathrm{I}^{\mathrm{\gamma}}\right],[\mathrm{w}],[\mathrm{y}] \mathrm{y}[\mathrm{k}]$, distribución complementaria con $\left[\mathrm{\gamma}^{\mathrm{j}}\right], \mathrm{y}$ variación libre restringida con [ü]].

En relación con la variable género, es posible afirmar que el comportamiento del género femenino es levemente superior al masculino, superándolo por 5 puntos porcentuales, con un total de $88 \%$ de realizaciones alofónicas.

El comportamiento respecto de la variable subsector también es con tendencia a la homogeneidad, aunque se presenta ligeramente superior en el subsector del valle del Queuco, el cual, con un $86 \%$ de realizaciones alofónicas, supera por 3 puntos porcentuales al sector Bío-Bío. 
Carolina Salinas Marchant, Gastón Salamanca Gutiérrez. Reanálisis de aspectos controversiales de la fonología segmental del chedungun hablado en Alto BioBío: el estatus fonémico del fono [४]

Así, finalmente, y en relación con la pregunta de investigación planteada: “¿Cuál

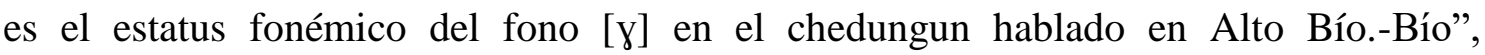
podemos afirmar que es un fonema: $/ y /$. Dicho fonema tiene como realizaciones

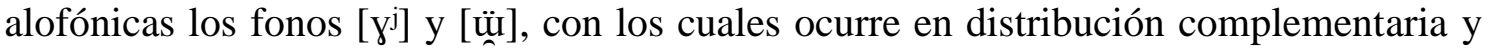
alternancia libre restringida, respectivamente. Por último, debemos señalar que el comportamiento de este segmento tiende a la homogeneidad cuando se consideran las variables género y subsector.

\section{Bibliografía}

Álvarez-Santullano, P. Descripción Fonológica del Huilliche, un dialecto del mapuche o araucano del centro-sur de Chile. Tesis de maestría no publicada. Universidad de Concepción, Concepción, Chile, (1986a). Impreso.

. "Descripción fonemática del huilliche; estudio comparativo". Alpha 2 (1986b): 25-50. Impreso.

Burquest, D. Análisis fonológico: Un enfoque funcional. Dallas: Summer Institute of Linguistics, 2009. Impreso.

Contreras, H. y Echeverría, M. “Araucanian Phonemics". IJAL 31. 2 (1965): 132-135.

Croese, R. "Estudio dialectológico del mapuche". Estudios Filológicos 15 (1980): 7-38.

Echeverría, M. Descripción fonológica del mapuche. Tesis de pregrado. Universidad de Concepción, 1963. Impreso. Impreso.

. "Descripción fonológica del mapuche actual". Boletín del Instituto de Filología de la Universidad de Chile 16 (1964):13-19. Impreso.

Fuentes, D. Caracterización articulatoria y acústica, en el contexto de un proceso de debilitamiento, de las realizaciones alofónicas de $/ j, w, \gamma, z, v, d /$ en el chedungun hablado en Alto Bío-Bío. Tesis de maestría no publicada. Universidad de Concepción, Concepción, Chile, 2015. Impreso.

Henríquez, M. Vitalidad fonológica del mapudungun en escolares mapuches pewenches y lafkenches de la VIII Región del Bío-Bío. Tesis para optar al grado de Doctor en Lingüística. Universidad de Concepción, 2013. Impreso.

Henríquez, M. y Salamanca, G. "Rasgos prominentes de la fonología segmental del chedungun hablado por escolares de Alto Bío-Bío". Alpha, 34 (2012): 153171.

Jiménez, M. Fonemas segmentales y realizaciones alofónicas del chedungún hablado en las comunidades de Trapa Trapa y Butalelbun, Alto Bío-Bío. Tesis para optar al grado de Licenciado en Educación con Mención en Español. Tesis no publicada. Universidad de Concepción, Concepción, Chile, 2014. Impreso.

Key, M. "Lingüística comparativa araucana". VICUS Cuadernos-Lingüística 2 (1978): 45-56. Impreso.

Lagos, D. "El estrato fónico del mapudungu(n)". Nueva Revista del Pacífico 19-20 (1981): 42-66. Impreso.

"Fonología del mapuche hablado en Victoria". Actas. Jornadas de Lengua y Literatura mapuche, (1984): 41-50. Impreso. 
Lenz, R. "Estudios Araucanos". Anales de la Universidad de Chile 90-98 (1895-1897). Impreso.

Mena, D. Frecuencia y distribución de vocales ensordecidas en el chedungun hablado por escolares del Alto Bío-Bío. Tesis para optar al grado de Licenciado en Educación con Mención en Español. Tesis no publicada. Universidad de Concepción, Concepción, Chile, (2013). Impreso.

Painequeo, H. El estatus fonológico de los segmentos (inter)dentales [t] [n] y [l] y el fono alveopalatal fricativo [S] en el sistema fonológico de la lengua mapuche del sector Budi, de la Región de la Araucanía, Chile. Tesis no publicada. Universidad de Concepción, Concepción, Chile, (2014). Impreso.

Pérez, Ch. Fonemas segmentales y alófonos del mapuzungun hablado en Curarrehue y determinación de los grafemas más idóneos para su representación. Tesis de maestría no publicada. Universidad de Concepción, Concepción, Chile, 2014. Impreso.

Pike, K. Phonemics: A Technique for Reducing Languages to Writing. Ann Arbor: The University of Michigan Press, 1947. Impreso.

Rivano, E. "Notes on the feature matrix for mapudungu vowels and related issues". RLA 28 (1990): 135-147. Impreso.

Sadowsky, S., Painequeo, H., Salamanca, G., Avelino, H. "Mapudungun”. Journal of the International Phonetic Association 43. 1(2013): 87-96. Impreso.

Salamanca, G. "Fonología del pehuenche hablado en Alto Bío-Bío". R.L.A., Revista de Lingüística Teórica y Aplicada 35 (1997): 113-124. Impreso.

Salamanca, G. y Quintrileo, E. "El mapuche hablado en Tirúa: Fonemas segmentales, fonotaxis y comparación con otras variedades". RLA 47.1 (2009):13-35. Impreso.

Salamanca, G., Cifuentes, E. y Figueroa, M. (2011). "Sistematización de criterios para la determinación de fonos, alófonos y formas básicas de los fonemas del español de Chile: una herramienta para la investigación y la docencia". Boletín de Filología, 46.2 (2011): 107-133. Impreso.

Salas, A. "Esbozo fonológico del mapudungu, lengua de los mapuches o araucanos de Chile central". Estudios Filológicos 11(1976): 143-153. Impreso.

-."Mapuche-Español. Análisis fonológico contrastivo". Vicus Cuadernos Lingüística 2 (1978): 57-85. Impreso.

. "Lingüística mapuche. Guía bibliográfica". Revista Andina, 10.2 (1992): 473537. Impreso.

El mapuche o araucano. Madrid: Mapfre, 2006. Impreso.

Salas, A. y Poblete, M. T. "Pares mínimos y estatus fonémico. ¿Causa o consecuencia?". RLA 35 (1997): 143-153. Impreso.

Sánchez, G. "Relatos orales en pewenche chileno". Estudios en honor de Yolando Pino Saavedra. Anales de la Universidad de Chile. 17 (1989): 289-360. Impreso.

Sánchez, M. Análisis fonético-fonológico segmental del mapudungun hablado en Lonquimay y determinación del inventario de grafemas más idóneos para su representación. Tesis de Magíster en Linguiística. Tesis no publicada. Universidad de Concepción, Concepción, Chile, 2014. Impreso.

Suárez, J. "The phonemes of an Araucanian dialect". IJAL 25(1959): 177- 181. Impreso. . "Reseña a Descripción fonológica del mapuche. IJAL 31(1964): 284-286. Impreso. 
Toro, S. Descripción fonológica del chedungún hablado en los sectores de Chenqueco y Chevquelavquen, Alto Bío-Bío. Tesis de Licenciado en Educación con Mención en Español. Tesis no publicada. Universidad de Concepción, Concepción, Chile, 2014. Impreso.

Trubetzkoy, N. Principios de fonología. Madrid: Cincel, [1939], 1987. Impreso. 